\title{
Epidemiology and risk stratification of minor head injuries in school-going children
}

Wing Yee Tong, ${ }^{1}{ }_{M R C P C H}$, Sek Wan $\underline{\text { Tan }},{ }^{2}{ }_{M R C P C H}$, Shu-Ling $\underline{\text { Chong }},{ }^{2,3}{ }_{M P H}$

\begin{abstract}
Introduction: Head injuries occur commonly in children and can lead to concussion injuries. We aim to describe the epidemiology of head injuries among school-going children and identify predictors of brain concussions in Singapore.

Methods: This is a retrospective study of children 7-16 years old who presented to the Emergency Department (ED) of KK Women's and Children's Hospital in Singapore with minor head injury between June 2017 and August 2018. Data including demographics, clinical presentation, ED and hospital management were collected using a standardised electronic template. Multivariable logistic regression analysis was performed to identify early predictors for brain concussion. Concussion symptoms were defined as persistent symptoms after admission, need for inpatient intervention, or physician concerns necessitating neuroimaging.

Results: Among 1,233 children (mean age, 6.6 years; $72.6 \%$ boys) analysed, the commonest mechanism was falls $(64.6 \%)$. Headache and vomiting were the most common presenting symptoms. A total of $395(32.0 \%)$ patients required admission, and $277(22.5 \%)$ had symptoms of concussion. Older age (13-16 years old) (adjusted odds ratio [aOR] 1.53, 95\% confidence interval [CI] 1.12-2.08), children involved in road traffic accidents (aOR 2.12, CI 1.17-3.85) and a presenting complaint of headache (aOR 2.64, CI 1.99-3.50) were significantly associated with symptoms of concussion.

Conclusion: This study provides a detailed description of the pattern of head injuries among school-going children in Singapore. High risk patients may require closer monitoring to detect post-concussion syndrome early.
\end{abstract}

Ann Acad Med Singap 2021;50:119-25

Keywords: Brain injuries, child, concussions, school, sport

\section{INTRODUCTION}

Head injuries are common childhood injuries that present to paediatric emergency departments (EDs). ${ }^{1}$ Falls are the most common cause in young children, while contact sports and road traffic injuries are common causes in school-going children. ${ }^{2}$ Majority of paediatric head injury cases are mild traumatic brain injuries (MTBI), defined as a Glasgow Coma Scale (GCS) of 14-15, among whom only a minority develop complications or require neurosurgical intervention. ${ }^{3,4}$ Nonetheless, given its prevalence and frequent presentation to healthcare facilities including EDs, minor head injuries constitute a significant clinical burden on the public healthcare system. ${ }^{5}$ Existing clinical prediction rules guide decision-making on which MTBI should receive a computed tomography (CT) of the brain. ${ }^{6,7}$

Among patients with MTBI, a subset may experience symptoms of concussion.

The 2017 Concussion in Sports group consensus statement defines concussion as "a traumatic brain injury induced by biomechanical forces", which typically results in a short-lived impairment of neurological function. It is also associated with acute clinical signs and symptoms, often with no abnormalities on standard neuroimaging studies. Resolution of clinical and cognitive features follows a sequential course, with most resolving within weeks, but some taking up to months. ${ }^{8}$

\footnotetext{
${ }^{1}$ Department of Paediatrics, KK Women and Children's Hospital, Singapore

${ }^{2}$ Department of Emergency Medicine, KK Women and Children's Hospital, Singapore

${ }^{3}$ Duke-NUS Medical School, Singapore

Correspondence: Dr Wing Yee Tong, Department of Paediatrics, KK Women's and Children's Hospital, 100 Bukit Timah Road, Singapore 229899.

Email: tong.wing.yee@singhealth.com.sg
} 
Growing evidence suggests that the long-term effects of concussion are under-recognised, and children are inadequately monitored following MTBI. Caregivers of children with minor head injuries have reported symptoms such as headaches, as well as attention and behavioural problems, persisting for months after mild head injury. ${ }^{9}$ Neurocognitive impairment has also been reported on objective testing during follow-up months after a concussion. ${ }^{10-12}$ It is also increasingly recognised that post-concussion syndrome (PCS), where children and adolescents experience persistent neurological, psychological and behavioural changes for at least 1-3 months after a concussion, ${ }^{13}$ is common among children with minor head injury, with studies reporting up to $29.3 \%$ of adolescents with mild traumatic brain injury who developed PCS. ${ }^{14,15}$

Minor head injuries among school-going children have not been reported in detail in Asia. Previous studies on paediatric head injuries in Singapore have focused on severe traumatic brain injury resulting in death or the need for neurosurgical intervention. ${ }^{15,16}$ Because of the lack of identification of children with concussive symptoms, children with MTBI are often discharged from the ED without outpatient follow-up. This results in a lack of formal evaluation, and an inability to quantify neurocognitive deficits.

In this study, we aim to study the epidemiology of minor head injuries among the school-going paediatric population. Our secondary aim is to understand, among children with minor head injuries, which child demonstrates symptoms of acute concussion, and would benefit from outpatient follow-up. We hypothesise that children between the ages of 13 and 16 years and children who are involved in contact sports have a high risk of concussion. The findings of this study would enable us to identify children with MTBI who require close follow-up and formal neurocognitive testing.

\section{METHODOLOGY}

\section{Study design}

This is a single-centre retrospective review of children between 7 and 16 years of age who presented to the ED of KK Women and Children's Hospital with head injury from June 2017 to August 2018. We included all children with the relevant International Classification of Diseases (ICD) codes for head injury that are currently enrolled into our electronic trauma surveillance registry, regardless of mechanism of injury. Head injury data (including the symptoms and signs post-injury) were recorded prospectively by the physician attending to the child during the patient encounter using the Electronic Health Record. This trauma registry is accredited by the Association for the Advancement of Automotive Medicine and is managed by a trauma coordinator.

\section{Inclusion and exclusion criteria}

We included all children between 7 and 16 years of age who presented to the ED within 24 hours of head injury. ${ }^{5}$ We also included hospitalised children and those who were discharged from the ED.

Children with a GCS of 13 or less at presentation were excluded, in keeping with the definition of a mild traumatic brain injury. Furthermore, children presenting with low GCS would necessitate resuscitation, and formed a different risk stratum of patients for whom close follow-up would already be required. Children requiring neurosurgical intervention, regardless of presenting GCS, were also excluded for the same reason as these would routinely be followed up after discharge from the hospital.

\section{Variables}

A standardised electronic template was filled up for every patient during his/her ED visit. We reviewed the mechanism, location, physical examination findings and further management, including neuroimaging, disposition and neurosurgical intervention (if any). We presented mechanism in the following categories: falls, road traffic accidents, sports-related injuries, interpersonal violence and others. Presenting symptoms included headache, vomiting, loss of consciousness, amnesia and contact seizures. Physical signs documented on examination included the GCS, any neurological deficits, signs of altered mental status, as well as any scalp haematoma, scalp laceration, skull fracture and basal skull fracture.

\section{Outcome measures}

For the secondary aim, we defined "concussion" as the following: persistent symptoms after admission including headache, nausea or vomiting $;^{8}$ need for intervention including the use of anti-emetics in the ward or physician concerns resulting in neuroimaging in the ED; or inpatient. Paediatric concussion assessment tools were not applied to the patients at the time of the study, as the data were retrospectively obtained from the surveillance registry.

\section{Analytical plan}

We described categorical variables using frequencies (and percentages) and continuous variables using 


\section{CLINICAL IMPACT}

\section{What is New}

- This study provides a detailed description of the pattern of head injuries in the school-going children in Singapore.

\section{Clinical Implications}

- In the busy emergency department setting where follow-up for all patients with head injury is not feasible, we identified risk factors for postconcussion syndrome in children who may benefit from closer monitoring.

means (with standard deviation, SD), or median (with interquartile ranges, IQR), depending on normality. For the primary aim, we presented the descriptive data by age strata. We chose to divide the study population into two groups of school-aged children-7-12 years old (local equivalent of elementary school) versus 13-16 years old (local equivalent of high school). We did so in view that different paediatric age groups are known to have head injuries from different causes; older students tend to get involved in high impact contact sports, and have anatomical and physiological differences compared to younger children (e.g. closure of bone physeal growth plates). The analysis for categorical variables was performed using chi square or Fisher's exact test, and the analysis for continuous variables using t-test, or Wilcoxon rank sum, depending on normality. For the secondary aim, we used a multivariable logistic regression model predicting for concussion, and present point estimates using odds ratios (ORs) and adjusted odds ratio (aORs) with their corresponding 95\% confidence intervals. Significance was taken at $P<0.05$. We used SPSS Statistics software version 26.0 for the analysis.

\section{RESULTS}

\section{Primary analysis}

There were a total of 1,233 children and adolescents with head injuries who were analysed, with 932 $(75.6 \%)$ under 13 years old. None of the children had prior neurosurgery performed and none had known underlying coagulopathy.

The commonest mechanism of injury in younger children (less than 13 years) was falls (64.6\%). Among the adolescents (13 to 16 years old), falls still comprised the majority of the head injuries $(52.2 \%)$, but sports injuries (13.3\%) and road traffic accidents (20\%) were relatively more common compared to the younger age group $(P<0.001)$ (Table 1$)$. Majority of the falls $(88 \%)$ occurred at ground level (when standing, running or walking) and were not falls from height. Sports that were associated with head injuries included basketball, soccer, judo, handball and cycling. Among all children and adolescents who were involved in road traffic accidents, 1,208 (97\%) were motor vehicle passengers, while the remaining minority were pedestrians, cyclists and motorcycle pillion riders.

Headache $(42.7 \%)$ was the most common symptom at presentation, and adolescents experienced this symptom more commonly than younger children under 13 years (52.4\% versus $40.3 \%, P \leq 0.001)$ (Table 1 ). Vomiting $(24.4 \%)$ was the second most common symptom, with a higher incidence in younger children compared with the adolescents $(25.3 \%$ vs $21.6 \%, P=0.191)$. Scalp haematoma $(24.8 \%)$ was the commonest physical finding in both children and adolescents $(26.5 \%$ vs $19.9 \%, P=0.023)$. The other less common physical examination findings noted in all age groups were scalp laceration $(3.6 \%)$, abnormal gait $(1.1 \%)$, and altered mental status $(0.4 \%)$. Eighteen patients $(1.5 \%)$ from the Children's ED underwent computed tomography (CT) brain scan, and a further 36 patients $(2.9 \%)$ underwent CT brain scan after inpatient admission at the discretion of the attending ward physician.

\section{Analysis of hospitalised patients}

Among the 395 children and adolescents who were hospitalised, $84(21.3 \%)$ of the patients had persistent symptoms during their stay. Most of them required the anti-emetic ondansetron $(229,58.0 \%)$, and $25(6.3 \%)$ required intravenous fluids. Among hospitalised patients, $357(90.4 \%)$ were monitored for less than 24 hours.

Of the total 1,233 patients, 277 (22.5\%) suffered concussion. The logistic regression model showed that adolescents 13 years or older (aOR1.53, 95\% confidence interval [CI] 1.12-2.08, $P<0.01)$, children involved in road traffic accidents (aOR 2.12, 95\% CI 1.17-3.85, $P=0.013$ ), and those with a presenting symptom of headache (aOR 2.64, 95\% CI 1.99-3.50, $P=<0.01)$ were significantly associated with symptoms of concussion. Gender (aOR 1.33, 95\% CI 0.91-1.67, $P=0.18$ ) and sports (aOR 1.39, 95\% CI 0.84-2.32) were not statistically significant for concussion.

\section{DISCUSSION}

This study provides a detailed description of the pattern of minor head injuries among the local paediatric 
Table 1. Demographics and characteristics of head injuries in children $(\mathrm{N}=1,233)$

\begin{tabular}{|c|c|c|c|c|}
\hline Results by age & & $\begin{array}{l}\text { Under } 13 \text { years }(\%) \\
(n=932)\end{array}$ & $\begin{array}{l}13 \text { to } 16 \text { years }(\%) \\
(n=301)\end{array}$ & $P$ value \\
\hline Gender & Male & $682(73.2)$ & $213(69.4)$ & 0.209 \\
\hline \multirow[t]{5}{*}{ Primary mechanism of injury } & & & & $<0.001$ \\
\hline & Fall & $640(68.7)$ & $157(52.2)$ & \\
\hline & Sports & $46(4.9)$ & $40(13.3)$ & \\
\hline & Road traffic collisions & $33(3.5)$ & $20(6.6)$ & \\
\hline & Others $^{\mathrm{a}}$ & $213(22.9)$ & $84(27.9)$ & \\
\hline \multicolumn{5}{|l|}{ Symptoms at presentation } \\
\hline & Difficult to arouse & $9(0.9)$ & $9(3.0)$ & 0.006 \\
\hline & Vomiting & $236(25.3)$ & $65(21.6)$ & 0.191 \\
\hline & Stiffening/limb jerking & $10(1.1)$ & $10(3.3)$ & 0.007 \\
\hline & Confused/disoriented & $19(2.0)$ & $17(5.6)$ & 0.001 \\
\hline & Headache & $372(40.3)$ & $155(52.4)$ & $<0.001$ \\
\hline & Amnesia & $45(4.9)$ & $42(14.2)$ & $<0.001$ \\
\hline \multicolumn{5}{|l|}{ Signs on physical examination } \\
\hline & Altered mental status & $3(0.3)$ & $3(1.0)$ & 0.145 \\
\hline & Focal neurological signs & $1(0.1)$ & $4(1.3)$ & 0.004 \\
\hline & Abnormal gait & $12(1.3)$ & $3(1.0)$ & $<0.001$ \\
\hline & Scalp haematoma & $246(26.5)$ & $60(19.9)$ & 0.023 \\
\hline & Scalp laceration & $38(4.1)$ & $11(3.7)$ & 0.739 \\
\hline & Signs of basal skull fracture & $6(0.6)$ & $2(0.7)$ & 0.972 \\
\hline
\end{tabular}

${ }^{a}$ Others include struck by high force object, interpersonal violence, machinery, tools/objects, foreign bodies, bites/stings

Table 2. Disposition and progress of hospitalised patients

\begin{tabular}{|c|c|c|c|}
\hline Results by age & Under 13 years $(\%)$ & 13 to 16 years $(\%)$ & $P$ value \\
\hline \multicolumn{4}{|l|}{ Disposition } \\
\hline Admitted & $258(28.2)$ & $137(46.6)$ & $<0.001$ \\
\hline Treated and discharged & $557(60.9)$ & $119(40.5)$ & \\
\hline Referred to specialist outpatient clinic (eg. neurosurgical clinic) & $93(10.2)$ & $36(12.2)$ & \\
\hline Referred to general practitioners, polyclinics & $5(0.5)$ & $1(0.3)$ & \\
\hline Absconded from Emergency Department & $2(0.2)$ & $1(0.3)$ & \\
\hline \multicolumn{4}{|l|}{ Progress of hospitalised patients } \\
\hline Total number hospitalised & $258(28.2)$ & $137(46.6)$ & \\
\hline Use of ondansetron & $158(61.2)$ & $71(51.8)$ & 0.071 \\
\hline Intravenous fluids & $17(6.6)$ & $8(5.8)$ & 0.771 \\
\hline Persistent symptoms & $58(22.5)$ & $26(19.0)$ & 0.418 \\
\hline
\end{tabular}


Table 3. Association of risk factors and concussion symptoms using univariate and multivariable regression models ( $\mathrm{n}=277$ )

\begin{tabular}{lllll}
\hline & & Univariate OR (95\% CI) & Multivariate OR (95\% CI) & P value (multivariate OR) \\
\hline Gender & Male & Reference & Reference & - \\
\hline & Female & $1.404(1.052-1.874)$ & $1.331(0.910-1.669)$ & 0.176 \\
\hline Age & & & Reference & $\mathbf{0 . 0 0 7}$ \\
\hline & Under 13 years old & Reference & $1.531(1.125-2.048)$ & $\mathbf{0 . 0 1 3}$ \\
\hline Mechanism of injury & 13 to 16 years & $1.738(1.296-2.322)$ & & 0.202 \\
\hline & Road traffic accident & $2.177(1.228-3.858)$ & $2.124(1.172-3.894)$ & $<2.393(0.838-2.316)$ \\
\hline
\end{tabular}

OR: odds ratio; CI: confidence interval

"Concussion symptoms" was defined as the following: (1) persistent symptoms after admission (including headache, nausea or vomiting), (2) need for intervention including the use of anti-emetics in the ward, or (3) physician concerns because of persistent symptoms resulting in neuroimaging in the emergency department or inpatient.

population of school-going age. Among children with minor head injuries, we found that the adolescent age group, children involved in road traffic accidents, and a presenting complaint of headache were significantly associated with symptoms of concussion.

Falls remain the commonest cause of head injury among Singapore school-going children. Sports-related head injuries are the next most common cause, with a higher incidence in the older age group (13 to 16 years), especially among boys. The increasing involvement of children and adolescents in sports, such as basketball and soccer in our study population, has resulted in an increasing incidence of minor head injuries. ${ }^{17}$ This highlights the importance of raising awareness of sports-related head injuries among parents, coaches and educators, so that rapid identification and treatment of head injuries can improve outcomes. In our model, sporting activities did not show a significant correlation with concussion symptoms, in contrast with previous studies..$^{18}$ A possible reason is that in the local setting, competitive contact sports such as rugby are less prevalent in school-going children compared to other countries. ${ }^{19}$ Furthermore, among those involved with contact sports, there may be relatively fewer that take part in highly competitive settings outside of the school context (e.g. inter-club competitions) where more severe injuries can occur.
It is well known that head injuries arising from road traffic accidents (RTAs) can be associated with severe traumatic brain injuries, and are more likely to require critical care or neurosurgical intervention. ${ }^{15}$ Our study findings demonstrate that minor head injuries caused by RTAs are also at increased risk of having concussion. Hence, even in children with seemingly "minor" symptoms after an RTA, it would be prudent for the clinician to educate parents about monitoring for persistent symptoms, and offer neurological follow-up for children with clinical concerns of concussion. The adolescent age group was found to be more likely to have concussion; the relationship between age and concussion was present even after accounting for mechanism of injury. We postulate that this could be related to the greater severity of injuries sustained in older children, as well as younger children being less able to self-report symptoms compared to adolescents.

This study supports headache in the ED as predictive of concussion. While headache is a commonly encountered symptom immediately after an MTBI, ${ }^{20}$ studies have shown that a substantial number of children continue to suffer from headaches months after the injury. ${ }^{21}$ Headache at initial evaluation after MTBI has previously been demonstrated be associated with PCS, ${ }^{22,23}$ although the actual incidence differs across studies, likely a result of survey methodology 
and the definition of persistent headache. Information on the expected course and duration of headaches after MTBI is vital when counselling injured children and their parents, and may prompt them to seek further evaluation should headaches persist or result in poorer academic performance and function.

Vomiting was a common presenting symptom in the ED. While clinically significant MTBI is also known to be uncommon in children who present with vomiting as a sole symptom, ${ }^{24}$ the discomfort triggered by headaches post-head injury can also trigger nausea and vomiting, confounding the analysis of vomiting as an isolated symptom of concussion. Given the complex relationship between vomiting and other symptoms of concussion, further studies are needed to determine if acute vomiting is indeed predictive of concussion.

Currently, there are no local protocols available on monitoring and assessment following minor head injuries. In the busy setting of the ED, time and manpower limitations make it impractical for the administration of lengthy screening cognitive tools for concussion. It is similarly unrealistic to offer all minor head injury patients outpatient follow-up to screen for persistent symptoms after a concussion. Our study has identified high-risk groups for concussion, and children with these factors can be identified in the ED setting and given appropriate discharge recommendations and follow-up, with objective neurocognitive testing to monitor for the development of PCS. This recommended approach may help to improve their long-term functional outcomes.

We acknowledge the limitations of the study. The study data were obtained retrospectively from a surveillance registry, hence details including a breakdown of the type of sport undertaken, or specific mechanisms of sporting injury, were not mandatory and only filled in some cases. As we do not routinely follow up children with MTBI, information which would have allowed better identification of children with symptoms of concussion, such as objective neurocognitive testing or screening for emotional and behavioural symptoms, could not be obtained. Furthermore, we were not able to accurately determine if the symptom of vomiting in children was due solely to concussion or could be attributed to other causes. We were also unable to determine the presence and duration of persistent symptoms beyond discharge of these children from the wards, as we did not have long-term follow-up data on the study population. Symptoms such as "headache" and "nausea" are often subjective and self-reported by patients. Studies in young athletes have shown that premorbid mood disorders and underlying psychiatric conditions may be associated with increased symptom reporting, ${ }^{25}$ which we were unable to account for in our ED setting. Hence, objective assessment tools will offer a more accurate assessment in future studies. Lastly, the data used in this study were collected from a single large paediatric tertiary hospital in Singapore. Future prospective multicentre studies are required to validate our findings on acute concussion symptoms following an MTBI.

\section{CONCLUSION}

This is the first study detailing the epidemiology of minor head injuries among school-going children in Singapore, which seeks to identify groups at risk of concussion. Further research is required to guide paediatricians in the recognition, management and follow-up of concussion, and to help develop protocols, including risk stratification of higher risk groups for formal neurocognitive testing.

\section{Acknowledgements}

The authors would like to acknowledge Ms Dianna Sri Dewi and Ms Jasmine Feng Xun Yi for their help in data collection.

\section{REFERENCES}

1. Faul $\mathrm{M}, \mathrm{Xu} \mathrm{L}$, Wald MW, Coronado VG. Traumatic brain injury in the United States: emergency department visits, hospitalizations and deaths 2002-2006. Atlanta (GA): Centers for Disease Control and Prevention, National Center for Injury Prevention and Control; 2010

2. Keenan HT, Susan LB. Epidemiology and outcomes of pediatric traumatic brain injury. Dev Neurosci 2006;28:256-63.

3. Quayle KS, Powell EC, Mahajan P, et al. Epidemiology of blunt head trauma in children in US emergency departments. N Engl J Med 2014;371:1945-7

4. Koepsell TD, Rivara FP, Vavilala MS, et al. Incidence and descriptive epidemiologic features of traumatic brain injury in King County, Washington. Pediatrics 2011;128:946-54.

5. Kuppermann N, Holmes JF, Dayan PS, et al. Identification of children at very low risk of clinically-important brain injuries after head trauma: a prospective cohort study. Lancet 2009;374:1160-70.

6. Lyttle, MD, Crowe, L, Oakley E, et al. Comparing CATCH, CHALICE and PECARN clinical decision rules for paediatric head injuries. Emerg Med J 2012;29:785-94.

7. Thiam DW, Yap SH, Chong SL. Clinical decision rules for paediatric minor head injury: are CT scans a necessary evil. Ann Acad Med Singap 2015;44:335-41.

8. McCrory P, Meeuwisse WH, Aubry M, et al. Consensus statement on concussion in sport - the 4th International Conference on Concussion in Sport held in Zurich, November 2012. Br J Sports Med 2013;47:250-8.

9. Blume H, Karameh H. Subacute concussion-related symptoms and postconcussion syndrome in pediatrics. Curr Opin Pediatr 2012;24:724-30 
10. Taylor HG, Dietrich A, Nuss K, et al. Post-concussive symptoms in children with mild traumatic brain injury. Neuropsychology 2010;24:148-59

11. Rabinowitz AR, Harvey S. Cognitive sequelae of traumatic brain injury. Psychiatr Clin North Am 2014;37:1-11.

12. Thomas DG, Collins MW, Saladino RA, et al. Identifying neurocognitive deficits in adolescents following concussion. Acad Emerg Med 2011;18:246-54.

13. Babcock L, Byczkowski T, Wade SL, et al. Predicting postconcussion syndrome after mild traumatic brain injury in children and adolescents who present to the emergency department. JAMA Pediatr 2013;167:156-61

14. Barlow KM, Crawford S, Stevenson A, et al. Epidemiology of postconcussion syndrome in pediatric mild traumatic brain injury. Pediatrics 2010;126:e374-81.

15. Chong SL, Chew SY, Feng JXY, et al. A prospective surveillance of paediatric head injuries in Singapore: a dual-centre study. BMJ Open 2016;6:e010618.

16. Chong SL, Liu N, Barbier S, et al. Predictive modelling in pediatric traumatic brain injury using machine learning. BMC Med Res Methodol 2015;15:22.

17. National Youth Council, Singapore. Youth statistics in brief 2019. Available at: https://www.nyc.gov.sg/en/initiatives/resources/youthstatistics-in-brief/. Accessed on 25 Apr 2020.
18. Kirkwood MW, Yeates KO, Wilson, et al. Pediatric sport-related concussion: a review of the clinical management of an oft-neglected population. Pediatrics 2006;117:1359-71.

19. Ministry of Culture, Community and Youth, Singapore. Sports Index 2015. Available at: https://www.sportsingapore.gov.sg/-/media/ SSC/Corporate/Files/About/Publications/Sports-Index-2015.pdf?1 $\mathrm{a}=\mathrm{en} \&$ hash $=\mathrm{F} 4 \mathrm{CF} 468 \mathrm{~F} 2 \mathrm{C} 02 \mathrm{E} 632172 \mathrm{CB} 1937 \mathrm{~F} 8 \mathrm{~A} 5384 \mathrm{~F} 6 \mathrm{E} 59692$. Accessed on 25 Apr 2020.

20. Eisenberg, Matthew A, William PM, et al. Duration and course of post-concussive symptoms. Pediatrics 2014;133:999-1006.

21. Blume HK, Vavilala MS, Jaffe KM, et al. Headache after pediatric traumatic brain injury: a cohort study. Pediatrics 2012;129:e31-9.

22. Zemek R, Barrowman N, Freedman SB, et al. Clinical risk score for persistent postconcussion symptoms among children with acute concussion in the ED. JAMA 2016;315:1014-25.

23. Choe MC, Heidi KB. Pediatric posttraumatic headache: a review. J Child Neurol 2016;31:76-85.

24. Dayan PS, Holmes JF, Atabaki S, et al. Association of traumatic brain injuries with vomiting in children with blunt head trauma. Ann Emerg Med 2014;63:657-65.

25. Morgan CD, Zuckerman SL, Lee YM, et al. Predictors of postconcussion syndrome after sports-related concussion in young athletes: a matched case-control study. J Neurosurg Pediatr 2015; 15:589-98. 